\title{
Editorial Welcome
}

$\mathrm{W}$ elcome to the first issue of our Sixth edition! Global Security and Intelligence Studies is a double-blind peer-reviewed academic journal that aims to bridge the two-way gap between academia and practitioners. We serve as common ground to a diverse and growing audience ranging from policymakers to academics to operators on the front lines. GSIS strives to provide work pertaining to the most current and relevant topics in an ever-evolving and rapidly expanding threat-scape. In order to keep up with the evolving field, we are excited to introduce a new peer-reviewed section to GSIS. The Critical Anal$y$ sis section will feature research subject to the same high academic standards as your other Research Articles but without the empirical requirement. This area of research is intended to showcase argumentative and explanatory research that stands on its own merits outside of a rigid experimental model. This edition sees the field further expand into epidemiology and the COVID-19 pandemic, the rise of political extremism in the US, corporate espionage, and how words have become weaponry. This issue covers a lot of ground in the security and intelligence industry!

We open this issue with an extremely timely discourse on extremism within the US. J.J. Brookhouser presents a quantitative analysis on domestic right-wing extremism and violence within the US from 2000 to 2020. Despite an increasing number of extremist groups with right-wing ideology, increases in violence associated with such groups have failed to be recognized as terrorism. Using the Global Terrorism Database Brookhouser correlated ideological motivators to attack lethality.

Our second research article in the issue Joshua Pease and James Hess depart of the research-rich field of extremist Islamism and look at their more moderate counterparts. The pair take an in-depth look at the philosophical underpinnings and limitations of Deobandi and Salafi doctrines. The research is founded on a very insightful qualitative meta-ethnography of past research and ethnographic accounts of the moderate (Deobandi and Salafi) and extremist (Taliban and Salafi Jihadi) doctrines. Applicable to researchers of Islamic-based terrorism the conclusions and methods of the authors also offer insight into the developmental evolution of budding extremist organizations in the US.

In our third article, Melissa Schnyder digs deeper into Europe's refugee crises by investigating how refugee protection and public support can be shaped. Her research articulates how participants from France and Germany perceive legislative protection measures when they are presented as aspects of national security. The results noted no significant differences for those presented with arguments centered on national security versus the control group who were not. The results 
suggest that four years of integration and contact with refugees have reduced intergroup fears.

Kadir Altintas rounds out our research section with an in-depth look at modern economic espionage. The research takes a detailed look at the structural and methodological differences in the transfer of technology for both industrial and corporate espionage. As cyber warfare and attacks continue to increase in effectiveness and accessibility while decreasing in cost the opportunity for non-state espionage for economic gain is also increasing. The research offers a methodological model, the Industrial-Corporate Espionage Pyramid, as a unifying model to explain instances of espionage.

We are excited to expand our peer-reviewed offerings to include non-empirically based critical analysis. As many of you are keenly aware the academic community at large is founded on direct reporting of qualitative and quantitative empirical processes delivered in a rigid Introduction-Methods-Results-Discussion/Conclusion format. This type of research is intended to offer replicable and familiar constructs for researchers to base future projects. It is the tried-andtrue basis of academic research, however limits scientific discourse in a way that hinders the promulgation of new ideas and theories. The unique aims and scope of GSIS to foster academic-practitioner discourse makes the move to broaden our research offerings a natural one. The new section, Critical Analysis, will offer non-empirically based research that presents new theories or discusses known topics at a higher academic level. Like other research articles presented within GSIS, the Critical Analysis section will offer double-blind peer-reviewed articles which represent the highest academic standards.

In our first Critical Analysis offering, Joshua Duke employs a Red Cell analytical examination of radical Islamic terrorist organizations as case studies on the phenomena. Looking into examples such as the Islamic State, the Haqqani Network, and al Qaeda, the author examines the factors that influenced the exportation of their unique forms of religious extremism. Drawing on these conclusions recommendations for counterterrorism strategies are discussed alongside the implications for national security and intelligence operations.

Margaret Marangione contributes to our Critical Analysis section with an in-depth discussion of information warfare and the global impact of its effects. Using disinformation campaigns identified and attributed to nations such as Russia and China the research discusses and outlines the cognitive effects that disinformation has on individuals and groups. The research highlights how nation-states at large have continued to exploit the modern hyper-connected world to achieve strategic objectives that were once only achievable through kinetic warfare.

We have two excellent policy offerings for this issue. In our first policy discussion, William Harlow discusses why Speech Communication is a vital, and an unfortunately overlooked, academic field in the security industry. His informative 
research offers analysts significant analytical insight into widely available intelligence. In our second article, Ardavan Khoshnood takes a deeper look into the assassination of the Iranian nuclear scientist Mohsen Fakhrizadeh. His research compares past Revolutionary Guard Corps tactics and assassinations to Fakhrizadeh's. His research outlines a detailed analysis pointing to severe counterintelligence failures from within the Revolutionary Guard. Both of these great discussions offer insights and practical applications into the vital use of open-sourced intelligence to inform security-based policy.

The COVID-19 pandemic became the center of not just everyday life in 2020. It also became the catalyst behind numerous conspiracy theories, anti-science narratives, memetic warfare, politically motivated rhetoric, and sadly, poor academic practices. In an effort to help clarify many of the false narratives surrounding COVID-19 and the pandemic, Cameron Carlson offers a detailed insight into the science of epidemiology and virology surrounding the pandemic.

Rounding out our spring edition are two fantastic book reviews. We are honored to feature a book review of the Russian language title Ordo Pluriversalis: The End of Pax Americana and the Rise of Multipolarity by Eugene Vertlieb. Additionally, Donald Meyerhoff offers an extremely timely review of Weaponized Words: The Strategic Role of Persuasion in Violent Radicalization and Counter-Radicalization.

Global Security and Intelligence Studies strives to be the source for research on global security and intelligence matters. As the global threat-scape evolves over time, GSIS is evolving to keep pace. The Journal is enhancing its academic edge, impact, and reach. We are working to build stronger bridges between senior leaders, academics, and practitioners. In addition to new content that advances the global discussion of security and intelligence, readers can anticipate more special issues with a focus on current security concerns.

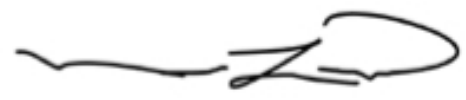

Melissa Layne, EdD

Editor-in-Chief

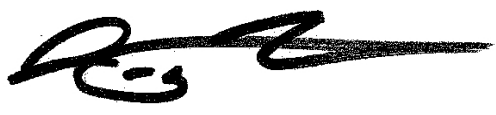

Carter Matherly, PhD

Associate Editor

\section{Carta editorial}

¡B ienvenidos al segundo número de nuestra quinta edición! Los estudios de seguridad e inteligencia globales se encuentran en la encrucijada entre la academia y los profesionales. Servimos a una audiencia diversa que va 
desde legisladores hasta operadores. En todo este espectro de lectores, GSIS se esfuerza por proporcionar trabajo relacionado con los temas más actuales y relevantes para que la seguridad y la inteligencia puedan avanzar tan rápido como las amenazas puedan adaptarse. Esta edición no es una excepción, nos complace ofrecer información sobre la pandemia COVID-19, discutir las operaciones cibernéticas en varios niveles, los impactos sociales y de seguridad de los cambios en la ecología, el papel de la religión en las ONG atacadas por el terror y cómo el encuadre puede influir percepción pública de las políticas de protección de refugiados. Cerramos esta edición con cinco reseñas de libros que abarcan desde operaciones psicológicas hasta la ciberseguridad. ¡Este problema cubre mucho terreno en la industria de la seguridad y la inteligencia!

Abrimos este número con un artículo orientado a las políticas de Margaret Marangione sobre la pandemia de COVID-19 que aborda abiertamente las deficiencias entre agencias y la necesidad de una mayor inteligencia en los campos de la bioseguridad y las amenazas biológicas. Trazando paralelismos con las fallas de inteligencia y políticas que llevaron a los ataques del 11 de septiembre, el perspicaz artículo destaca fallas similares que, combinadas con una política deficiente, no específica de ninguna administración, no brindaron protección y preparación. Aunque no es un término o disciplina común en la actualidad, MEDINT (inteligencia médica) debería ser un lugar común en la comunidad de inteligencia.

Al Lewis explora los conceptos de la teoría de la guerra justa y cómo las capacidades cibernéticas se están empleando en un marco de jus ad vim (poco antes de la guerra) mientras se logran efectos que generalmente se ven durante los conflictos de guerra. El autor destaca la falta de un marco ético para guiar la guerra cibernética similar a la teoría de la guerra justa, que ofrece la base fundamental que describe el uso ético del poder bélico tradicional.

Parecido a la contaminación del río Flint de 2014 en Flint, Michigan, el New River Report, de Kristin Drexler, refleja impactos similares en el sistema socioecológico. A través de las entrevistas de Drexler con los residentes de las comunidades ribereñas, se refleja la misma falta de confianza en la industria y el gobierno, sentimientos de impotencia y futuros inciertos de quienes viven en las comunidades de Flint. A partir de las entrevistas, el autor identifica áreas dentro de nuestro sistema socioecológico donde los contaminantes antropogénicos representarán una amenaza a largo plazo y perjudicial, como la salud humana, los medios de vida, el medio ambiente, la cultura y la justicia social. El autor ofrece posibles soluciones a este problema a nivel nacional para incluir discusiones entre la industria, el gobierno, la agricultura y los ciudadanos de la comunidad.

En el artículo ONG internacionales atacadas por el terror: el impacto de la religiosidad en la independencia, la neutralidad y la imparcialidad, la autora, Dra. Kathryn Lambert, explora el impacto que tiene la fe religiosa en los principios humanitarios básicos y la defensa de problemas entre las organizaciones 
humanitarias seculares y religiosas que han sido atacado por organizaciones terroristas. Su estudio longitudinal abarca dieciocho años y 92 organizaciones. En el complejo mundo actual de seguridad y amenazas, las organizaciones humanitarias llegarán rápidamente a la escena o al teatro con el objetivo de ayudar a su causa, independientemente de las complejidades que traen consigo. Comprender estas complejidades es fundamental para el comandante o líder de ayuda humanitaria y de socorro. El Dr. Lambert ofrece una visión única y crítica de estos efectos.

En una época política que ha visto una retórica cada vez más divisiva, la Dra. Melissa Schnyder ofrece una visión experimental de cómo el encuadre puede afectar las dicotomías dentro del grupo fuera del grupo. Utilizando poblaciones en Francia y Alemania, el autor señala que solo algunos marcos son efectivos cuando se intenta obtener apoyo para los migrantes refugiados. Esta investigación es fundamental para los profesionales de inteligencia y seguridad en las disciplinas de inmigración y seguridad nacional y podría ofrecer información y señales sobre los intentos de influencia de un actor extranjero.

El Dr. Jim Burch completa nuestros artículos de investigación para este número con una discusión sobre cómo operacionalizar la comunidad de inteligencia. En una era de guerra donde términos como Inteligencia Artificial, operaciones conjuntas de múltiples dominios y enemigos adaptables ágiles enmarcan la mentalidad del guerrero, ¿qué se puede decir sobre la recopilación de inteligencia? El Dr. Burch utiliza el marco de seis facetas de Hesselbeim para la transformación a fin de poner en funcionamiento y cerrar brechas críticas en los esfuerzos de recopilación de inteligencia.

En nuestro segundo artículo sobre seguridad cibernética, el Dr. Douglas Rose ofrece una visión técnicamente avanzada del futuro de la guerra y la teoría cibernéticas. Imagínese si la física avanzada y el análisis estadístico se combinaran para crear un dominio físico, pero virtual. Los espacios discursivos resultantes ofrecen mapas en los que se pueden realizar operaciones de inteligencia, pero en una era que tiene a la Inteligencia Artificial y el Aprendizaje Automático como la próxima gran evolución, esta investigación pregunta quién o qué está monitoreando los fractales ocultos. Las teorías e ideas propuestas en este artículo son fácilmente un cambio de paradigma en la guerra cibernética y la inteligencia.

Nuestros autores se han mantenido extremadamente ocupados durante los últimos meses secuestrados en condiciones de cuarentena. Esto les ha dado un tiempo considerable para ponerse al día con una lectura ligera. Como resultado, nuestra bandeja de entrada estaba repleta de reseñas de libros y estamos emocionados de presentar cuatro de los mejores con esta edición. El Dr. Mark Peters II ofrece una profunda visión y perspectiva del controvertido libro de Chris Wylie, Mindf* ck, Cambridge Analytica y The Plot to Break America. Con la temporada de elecciones en marcha, esta revisión no podría ser más oportuna o relevante que la actual. La Dra. Elise Rainer nos trae una revisión y una recomendación 
que invita a la reflexión para Porque somos humanos: impugnando el apoyo de los Estados Unidos a los derechos humanos de género y sexualidad en el extranjero. Los estudiantes, profesionales y académicos de derechos humanos y LGBTI se beneficiarían de esta revisión y libro autorizado sobre un grupo vulnerable que aún es perseguido en otras naciones. Alfred Lewis nos lleva de regreso al dominio cibernético con su reseña de El hacker y el estado: ataques cibernéticos y la nueva normalidad de la geopolítica. Un área creciente de jerga técnica y complejidad cada vez mayores The Hacker and the State ofrece una presentación y discusión digeribles en un formato no técnico. En nuestra revisión final del libro, el Dr. Jim Burch analiza Los innovadores: cómo un grupo de hackers, genios y geeks crearon la revolución digital. En parte relato histórico, en parte liderazgo y en parte destreza en equipo, este libro es una lectura obligada para cualquiera que trabaje en el dominio cibernético hoy.

Estudios de inteligencia y seguridad global se esfuerza por ser la fuente de investigación sobre asuntos de inteligencia y seguridad global. A medida que el panorama de amenazas global evoluciona con el tiempo, GSIS evoluciona para mantener el ritmo. La revista está mejorando su alcance académico, su impacto y su alcance. Estamos trabajando para construir puentes más sólidos entre líderes senior, académicos y profesionales. Además del contenido nuevo que avanza en la discusión global sobre seguridad e inteligencia, los lectores pueden anticipar problemas más especiales centrándose en las preocupaciones de seguridad actuales.

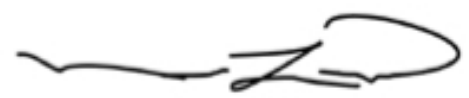

Melissa Layne, EdD Editora Principal

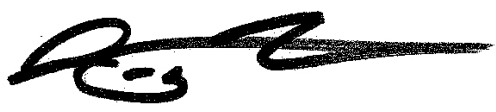

Carter Matherly, $\mathrm{PhD}$ Editor Asociado

\section{编者按}

欢迎来到第六版的第一期！GSIS 是一本经同行评审的双盲学术期刊，旨 在弥合学术界和从业者之间的双向鸿沟 从决策者到学术界再到一线运营 商 我们为越来越多的受众提供了共同的基础。GSIS 致力于在不断发展和 迅速扩展的威胁领域提供与最新和最相关主题相关的工作 为了跟上不断发 展的领域 我们很高兴为 GSIS。引入一个新的经过同行评审的部分 关键 分析部分将以与您其他研究文章相同的高学术标准为研究重点 但没有经验 要求 该研究领域旨在展示辩论性研究和解释性研究 这些研究在严格的实 验模型之外还具有自己的优点 在此版本中 该领域进一步扩展到流行病学 
和。COVID-19 大流行, 美国政治极端主义的抬头 公司间谍活动以及言语 如何成为武器 该问题涵盖了安全和情报行业的许多领域!

我们以非常及时的关于美国内部极端主义的论述来打开这个问题 （J. J. Brookhourser）对 2000 年至 2020。年美国国内右翼极端主义和暴力行为 进行了定量分析 尽管有右翼意识形态的极端主义团体数量不断增加，但与 此类团体相关的暴力活动并未被视为恐怖主义。使用全球恐怖主义数据库 布鲁克豪斯关联了意识形态动机来打击杀伤力。

我们在本期（John Pease）和（James Hess）中发表的第二篇研究文章脱 离了研究广泛的极端伊斯兰主义领域 并着眼于较为温和的同行。两人深入 研究了迪奥班迪和萨拉菲学说的哲学基础和局限性。该研究建立在非常有 见地的定性元民族志学基础之上 该元民族志学涵盖了中度 (Deobandi and Salafi）和极端主义（Taliban and Salafi Jihadi）教义的以往研究和人 种学研究。作者的结论和方法适用于基于伊斯兰的恐怖主义的研究人员 还 提供了对美国萌芽的极端主义组织的发展演变的深刻见解。

在第三篇文章中 梅利莎 施耐德（Melissa Schnyder）通过研究如何形成 难民保护和公众支持的方式 更深入地研究了欧洲的难民危机。她的研究阐 明了来自法国和德国的参与者在被视为国家安全方面时如何看待立法保护 措施。结果表明 那些以国家安全为中心的论点与没有以国家安全为中心的 论点没有明显差异。结果表明 四年的融合和与难民的接触减少了群体间的 恐惧。

Kadir Altintas 通过深入研究现代经济间谍活动来完善我们的研究部分。 这项研究详细研究了工业和企业间谍活动中技术转让的结构和方法上的差 异。随着网络战和攻击在降低成本的同时继续提高有效性和可访问性 为 经济利益而进行非国家间谍活动的机会也在增加。该研究提供了一种方法 模型 即工业公司间谍活动金字塔 作为解释间谋活动实例的统一模型。

我们很高兴将我们的同行评审产品扩展到包括非基于经验的关键分析。你 们中许多人都敏锐地意识到 整个学术界都建立在以有序介绍 方法 结果 讨论/结论格式交付的定性和定量经验过程的直接报告基础上。这种类型 的研究旨在为研究人员提供可复制且熟悉的结构 以作为未来项目的基础。 它是学术研究的久经考验的真实基础 但是以某种方式限制了科学话语 从 而阻碍了新思想和新理论的颁布。GSIS 促进学术从业人员话语的独特目标 和范围。使扩大我们的研究范围成为自然而然的举动。新的 “关键分析” 部分将提供基于非经验的研究 以提出新的理论或在更高的学术水平上讨 论已知的主题。像 GSIS 中提供的其他研究文章一样 “关键分析” 部分将 提供代表最高学术标准的双盲同行评审文章。

在我们的第一个批判分析产品中约书亚 杜克（Joshua Duke）使用红细胞 对激进的伊斯兰恐怖组织进行分析检查 作为对该现象的案例研究。通过研 究诸如伊斯兰国 哈卡尼网络和基地组织等例子 作者研究了影响其独特形 
式的宗教极端主义输出的因素。根据这些结论 讨论了对反恐战略的表彰以 及对国家安全和情报行动的影响。

玛格丽特 马兰吉欧内（Margaret Marangione） 通过对信息战及其影响 的全球影响进行了深入讨论, 为我们的关键分析部分做出了贡献。通过使 用被识别并归因于俄罗斯和中国等国家的虚假信息运动, 该研究讨论并概 述了虚假信息对个人和群体的认知作用。这项研究突出了整个民族国家如 何继续利用现代高度互联的世界来实现曾经只能通过动战来实现的战略目 标。

对于此问题, 我们提供了两种出色的策略。在我们的第一次政策讨论中, 威廉 哈洛 (William Harlow) 讨论了为什么语音通信在安全行业中是至 关重要的且不幸地被忽视的学术领域。他的信息丰富的研究为分析师提 供了对广泛可用情报的重要分析见识。在我们的第二篇文章中, 阿达万 科什诺德 (Ardavan Khoshnood) 更深入地研究了伊朗核科学家 (Mohsen Fakhrizadeh） 被暗杀的情况。他的研究将革命卫队的过去战术和暗杀行 为与法赫扎迪 (Fakhrizadeh) 的策略进行了比较。他的研究概述了详细 的分析, 指出革命卫队内部严重的反情报失败。这两场精彩的讨论都为深 入了解开源智能以告知基于安全的策略提供了见识和实际应用。

COVID-19 大流行不仅在 2020 年成为日常生活的中心。它还成为众多阴谋 理论, 反科学叙事, 模因战争, 出于政治动机的言论以及令人遗憾的不良 学术实践的催化剂。为了帮助弄清围绕 COVID-19 和大流行的许多错误叙 述（Cameron Carlson）提供了有关大流行周围的流行病学和病毒学的详细 见解。

完善我们的春季版是两个很棒的书评。我们很荣幸为您介绍尤金 韦特 利布 (Eugene Vertlieb) 撰写的俄文标题 Ordo Pluriversalis: 美国 Pax 的终结与多极化的崛起》的书评。此外, 唐纳德 梅耶霍夫 (Donald Meyerhoff）极为及时地回顾了武器化词语：说服在暴力激进和反激进中 的战略作用。

全球安全和情报研究致力于成为全球安全和情报问题研究的来源。随着全 球威胁形势的发展, GSIS 也在不断发展。该期刊正在增强其学术优势, 影响力和影响力。我们正在努力在高级领导人, 学者和从业者之间建立更 牢固的桥梁。除了可以推动全球安全和情报讨论的新内容外, 读者还可以 预期更多特殊问题, 重点关注当前的安全问题

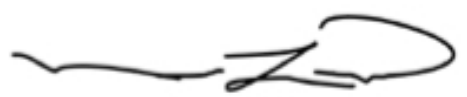

教育学博士主编

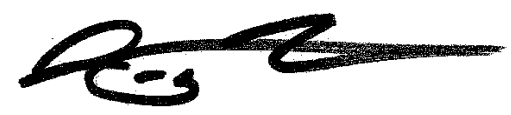

博士副主编 